\title{
Acoustic Vortices with High-order Orbital Angular Momentum by a Continuously Tunable Metasurface
}

\author{
Shi-Wang Fan, ${ }^{1,2}$ Yan-Feng Wang, ${ }^{3}$ Liyun Cao, ${ }^{2}$ Yifan Zhu, ${ }^{2}$ A-Li Chen, ${ }_{1}^{1}$ Brice Vincent, ${ }^{2}$ \\ Badreddine Assouar, ${ }^{2, *}$ Yue-Sheng Wang, ${ }^{1,3, *}$ \\ ${ }^{1}$ Institute of Engineering Mechanics, Beijing Jiaotong University, Beijing, 100044, China \\ ${ }^{2}$ Institut Jean Lamour, CNRS, Université de Lorraine, Nancy, 54000, France \\ ${ }^{3}$ Department of Mechanics, School of Mechanical Engineering, Tianjin University, Tianjin, 300350, China
}

\begin{abstract}
Metasurfaces-based acoustic vortex generators formed by fixed microstructures can only transfer the first-order orbital angular momentum (OAM) at a single frequency in a waveguide. Here, the multiple order acoustic vortices are realized through a helical matesurface design which is geometrically compact, broadband and tunable. The proposed metasurface consists of matched helixes rotating into the corresponding helical channels allowing to modulate the reflected phase shifts of the individual units. The units are arranged in a compact circular configuration, and then passively transforming an incident plane wavefront into the desired spiral wavefront with a controllable topological charge. The reported theoretical, numerical and experimental results evidence that multiple orders of OAM modes can be continuously generated by using only a single metasurface. The tunable vortices design is relevant to the pragmatic applications of metasurfaces in real-time acoustic communications, spanners and tweezers.
\end{abstract}

Keywords: acoustic vortices, broadband tunable metasurfaces, high-order orbital angular momentum

\section{Corresponding authors:}

*badreddine.assouar@univ-lorraine.fr

*yswang@tju.edu.cn 
Acoustic metasurfaces (AMs) are constituted by rationally designed individual units with preconceived phase and amplitude responses to efficiently modulate wavefronts. ${ }^{1,2}$ However, there still is one particularly technical difficulty to broaden and tune the operating frequencies. ${ }^{3,4}$ Indeed, most of the existing AMs have fixed and rigid microstructures that can only operate at a single or a narrow frequency range. To break through this restriction, a few effective ways have been proposed to design integrally adjustable $\mathrm{AM}$ structures $^{5,6}$ or independently reconfigurable units of AMs. ${ }^{7-15}$ These works of tunable design have been explored to deal with multiple acoustic functions including asymmetric transmission, ${ }^{5-7}$ sound shaping, ${ }^{8,9}$ acoustic focusing, ${ }^{10,11}$ flat reflected manipulation, ${ }^{12,13}$ curved cloaking and illusion, ${ }^{14,15}$ and so on.

Among various functions of AMs, acoustic vortex (AV) is one of the most promising applications to transfer orbital angular momentum (OAM) for micro-fluidic mixing, ${ }^{16}$ high-speed communication, ${ }^{17}$ acoustic tweezers or spanners, ${ }^{18,}{ }^{19}$ etc. The vortex beam is characterized by a spiral pressure wavefront $\exp (\operatorname{im} \theta)$, where the phase factor $\phi=m \theta$ is linearly proportional to the azimuth angle $\theta$. The integer $m$ indicates the topological charge (TC), corresponding to the OAM order of the vortex carried, which determines the total phase accumulated in one full annular loop around the propagation axis. ${ }^{20-24}$ Through the designed AMs, AVs can be produced in an infinite free space, ${ }^{25,26}$ or in a waveguide system. ${ }^{27-30}$ The vortices in free space can eliminate the boundary effect and maintain the OAM pattern in the near field. However, due to the divergence of the radiation cone 
angle, the pressure amplitude of the AV decreases and the pressure profile expands with the propagation distance increasing. Then, the vortex signal is severely limited by its transmitted distance in the infinite free space. Also, AVs generated in a waveguide system with a suitable wave number might be more beneficial to the remote signal propagation. Recently, the Helmholtz resonators were utilized to get AVs, and reduce the AMs thickness down to $1 / 2$ wavelength. ${ }^{27,28} \mathrm{~A}$ coiling-space AM was designed with $1 / 3$ wavelength. ${ }^{29}$ Due to the fixed microstructures, they were only used to generate first-order AVs at a single frequency.

In this letter, we introduce the matched helical mechanism ${ }^{10-12}$ to design a continuously tunable acoustic metasurface (TAM). By changing a helical depth, we can dynamically manipulate the reflected phase shifts of the acoustic wave from the TAM unit. Then, the higher-order AVs can be achieved. The field amplitude and phase distributions of the higher-order AVs are demonstrated through both analysis and simulation. Experiments are further carried out to evidence the realization of the multiple OAM states by the reconfiguration of only one TAM. In addition, the helical design of the TAM can decrease its thickness down to less than $1 / 4$ wavelength at a relatively low frequency. 
(a)

(b)
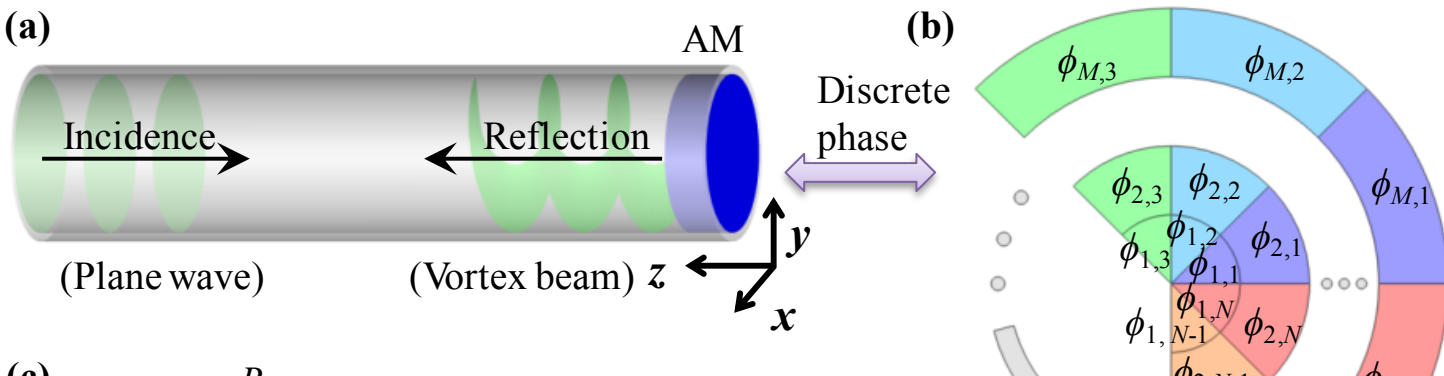

(c)
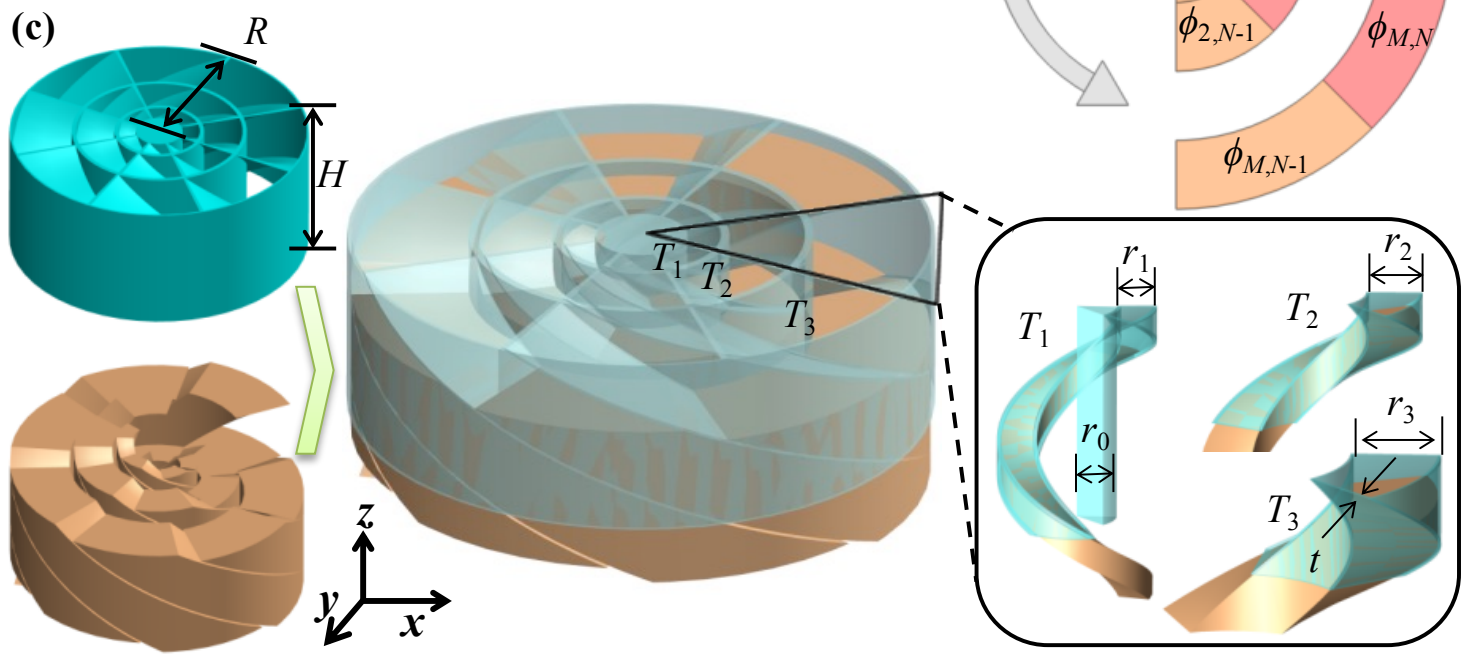

FIG. 1. (a) The schematic diagram of the realization of acoustic vortex in a cylindrical waveguide. Wave front shapes are shown in green. (b) Simplified diagram of the AM with discrete phase shifts of $2 m \pi$. (c) The designed TAM with individually adjustable sectors consisting of a matched helix (brown) rotated into a helical channel (blue) from bottom to top. The insets show the geometric details of the tunable units.

Figure 1(a) shows the case of an acoustic plane wave which is incident on an AM, and then reflected as a vortex beam from its surface. The AM consists of $M$ rows in the radial direction, in which each row of the planar layer is divided into $N$ sectors, as shown in Fig. 1(b). To achieve the AVs with a TC of the integer value $m$, the proposed circular configuration should cover the phase shift range of $2 m \pi$ with the same phase gradient. Then, the desired phase shift reflected from the $j$-th sector on each row is given by 


$$
\begin{aligned}
& \phi_{M, N}=2 \pi m(j-1) / N, \\
& (j=1,2, \cdots, N), \\
& N>\operatorname{Max}(2|m|+1,4) .
\end{aligned}
$$

In this general configuration, $N$ controls the resolution of the generated helical wavefront and determines the maximum TC that the AM can produce, while $M$ controls the amount of the reflected power.

For theoretical interpretation, in order to generate AVs in a rigid waveguide tube with the radius of $R$, the reflected field can be represented as the superposition of cylindrical Bessel modes:

$$
p_{m, n}=\exp (-i \omega t) \sum_{m} \sum_{n} A_{m, n} J_{m}\left(k_{m, n} R\right) \exp \left[i\left(m \theta+k_{z} z\right)\right]
$$

where the axial wave number is $k_{z}^{2}=k^{2}-k_{m, n}{ }^{2} ; k=2 \pi / \lambda$ is the wave number, in which $\lambda=c_{0} / f$ is the wavelength with $f$ being the incident frequency and $c_{0}$ the sound velocity; $k_{m, n}$ represents the radial wave number which is the $n$th positive root of the equation $d J_{m}\left(k_{m, n} R\right) / d\left(k_{m, n} R\right)=0 ; J_{m}\left(k_{m, n} R\right)$ is the $m$-order Bessel function; and $A_{m, n}$ is the modal amplitude.

Table 1. The propagable wave numbers (limiting frequencies $f_{m, n}$ ) for the fundamental modes in the cylindrical waveguide with diameter $R=7 \mathrm{~cm}$.

\begin{tabular}{lll}
\hline & $k_{m, 1} R\left(f_{m, 1}\right)$ & $k_{m, 2} R\left(f_{m, 2}\right)$ \\
\hline$m=1$ & $1.84(1.44 \mathrm{kHz})$ & $5.33(4.16 \mathrm{kHz})$ \\
$m=2$ & $3.05(2.38 \mathrm{kHz})$ & $6.71(5.23 \mathrm{kHz})$ \\
$m=3$ & $4.20(3.28 \mathrm{kHz})$ & $8.02(6.25 \mathrm{kHz})$ \\
$m=4$ & $5.32(4.15 \mathrm{kHz})$ & $9.28(7.24 \mathrm{kHz})$ \\
\hline
\end{tabular}

We construct the compact TAM as an assembly of eight fanlike sections $(N=8)$ of helical channels over the whole azimuth, as shown in Fig.1(c). This number of sections gives a reasonably good 
resolution for generating vortices with $\mathrm{TC}$ of $|m| \leq 3$ according to Eq. (1). For generating more higher-order vortex beams or finer resolutions, the number of sections can be increased accordingly.

Each section is designed to be composed of three rows $(M=3)$ of helical channels in the radius (more rows can be employed for a larger radius). The total diameter of the cylindrical TAM is $R=7$ cm. By considering Eq. (2), the corresponding propagable wave numbers and the limiting frequencies for the fundamental mode are listed in the Table 1. The other modes gradually fall in the evanescent state and are finally captured in the near field of the surface at low frequencies, while the vortex fields will be disturbed by the superposition of higher-order Bessel-like modes at high frequencies, see the details in supplementary material.

From Table 1, we can know that the first, second and third order vortices can be generated at the frequency ranges of $[1.44,4.16],[2.38,5.23]$ and $[3.28,6.25] \mathrm{kHz}$, respectively. These three AVs can be produced in the intersection frequency ranges as $[3.28,4.16] \mathrm{kHz}$. However, there is no common frequency range between them when $m \geq 4$. Therefore, the continuously frequency range considered in the present work will be from 1.5 to $6.0 \mathrm{kHz}$.

The designed TAM consists of matched helixes rotated into helical channels from bottom to top, as shown in Fig. 1(c). Based on the matched helical mechanism, ${ }^{10-12}$ when the matched helix is tuned by rotating, the channel length characterized by the helical depth $(h)$ will be changed. Each sector in each row can be regarded as an independent tunable unit. The reflected phase shift can be then varied 
by changing the helical depth to in real-time generate multiple order AVs in broadband frequency range. The total height of the TAM is $H=55 \mathrm{~mm}$. The three rows have the radius of $r_{1}=10 \mathrm{~mm}$, $r_{2}=20 \mathrm{~mm}$ and $r_{3}=30 \mathrm{~mm}$, respectively. The thread leads in three rows are $T_{1}=70 \mathrm{~mm}, T_{2}=130 \mathrm{~mm}$ and $T_{3}=240 \mathrm{~mm}$, respectively. The unit wall and blade thickness are $t=1 \mathrm{~mm}$. The thin total thickness of the TAM, the easy tunable operation of the unit, and the plane wavefront reflected from the helical channels can be simultaneously satisfied with tradeoffs between three parameters: the thread lead, the helical radius, and the central column, in the limit of approximate equality. The central column radius is $r_{0}=10 \mathrm{~mm}$. To ensure the computational accuracy of the reflected field, the center column will be considered in the first row in the following calculations. 
(a) Theoretical model $\left(T_{1}\right)$

(c) Theoretical analysis $\left(T_{1}\right)$ (d) Numerical simulation $\left(T_{1}\right)$
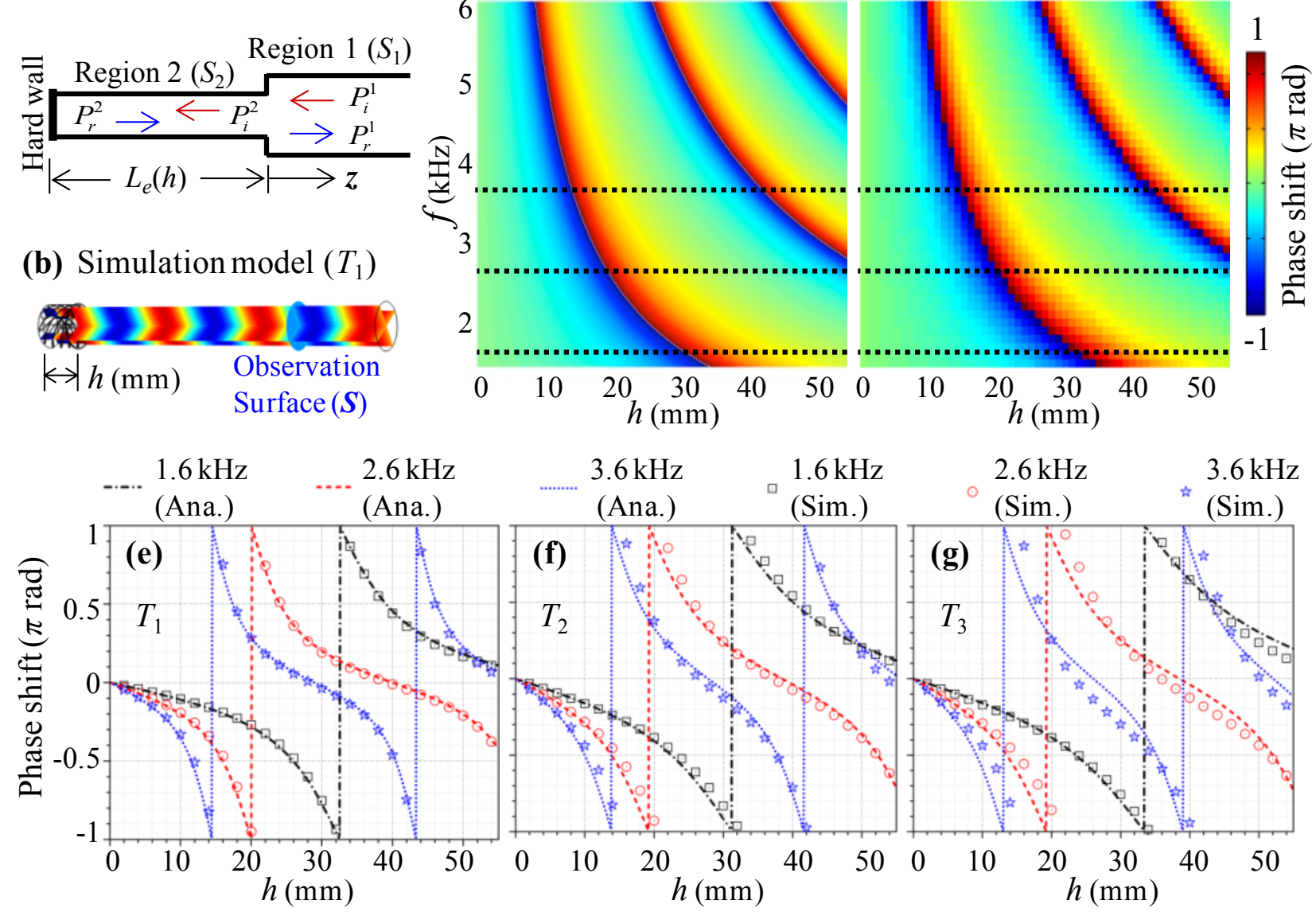

FIG. 2. (a) The equivalent theoretical model and (b) the numerical simulation model of the helical units for the

first-row of the designed TAM. The reflected phase shifts of (c) the theoretical analysis and (d) the numerical simulation. (e) The cut lines of the phase responds at the frequencies of 1.6, 2.6 and $3.6 \mathrm{kHz}$. The variation of the phase shift with the helical depth for (f) the second-row and (g) the third-row units at the three frequencies.

To illustrate the broadband tunability, we should first obtain the relation between the phase shift and the helical depth in a continuous frequency domain. We present the computing process for the first-row $\left(T_{1}\right)$ as an example. Then, the equivalent propagation path $\left[L_{e}(h)\right]$ of the theoretical model is developed in Fig. 2(a) to calculate the phase shifts by using the transfer matrix method. ${ }^{14}$ Therefore, the normalized amplitude and phase shift of reflection can be calculated by 


$$
p_{r}=A \exp (i \phi)=\frac{S_{12}+\exp \left[-2 i k L_{e}(h)\right]}{1+S_{12} \exp \left[-2 i k L_{e}(h)\right.},
$$

where the area ratio is $S_{12}=\left(S_{1}-S_{2}\right) /\left(S_{1}+S_{2}\right)$. It can be found from Eq. (3) that the reflected amplitude $\left[A=\operatorname{abs}\left(p_{r}\right)\right]$ is always equal to 1 by MATLAB programming calculation. This means total reflection from the TAM without any energy loss. Variation of the phase shift $\left[\phi=\arg \left(p_{r}\right)\right]$ with the incident frequency and helical depth for the theoretical model is calculated and illustrated in Fig. 2(c). We refer to supplementary material for the theoretical derivations and simulated settings in detail.

Then, the finite element model for the helical units in the first-row is built in Fig. 2(b) by using the commercial software COMSOL Multiphysics 5.4. The simulated phase shift responses for different frequencies and helical depths are shown in Fig. 2(d).

For quantitative comparison with considering the limited frequencies in Table 1, the cut lines of the phase shift at the frequencies of 1.6, 2.6 and $3.6 \mathrm{kHz}$ [dashed lines in Figs. 2(c) and 2(d)] are plotted in Fig. 2(e). Similarly, we could get the phase shifts for the other two-rows $\left(T_{2}\right.$ and $\left.T_{3}\right)$. The corresponding theoretical and simulated results are shown in Figs. 2(f) and 2(g), respectively, see supplementary material.

It is observed that the theoretical and numerical results for these three-rows are consistent with each other in terms of the number of full $2 \pi$ ranges and the varying phase shift trend. Therefore, the reflected acoustic waves can be manipulated broadband by continuously adjusting the helical depth. All the needed phase values by Eq. (1) and the corresponding helical depths at an operational 
frequency can be obtained directly from the phase shift responses. Note that the interactions between units are very weak and negligible, see supplementary material. For clarity, the tuned helical depths for multiple OAM modes at different frequencies are listed in supplementary material.
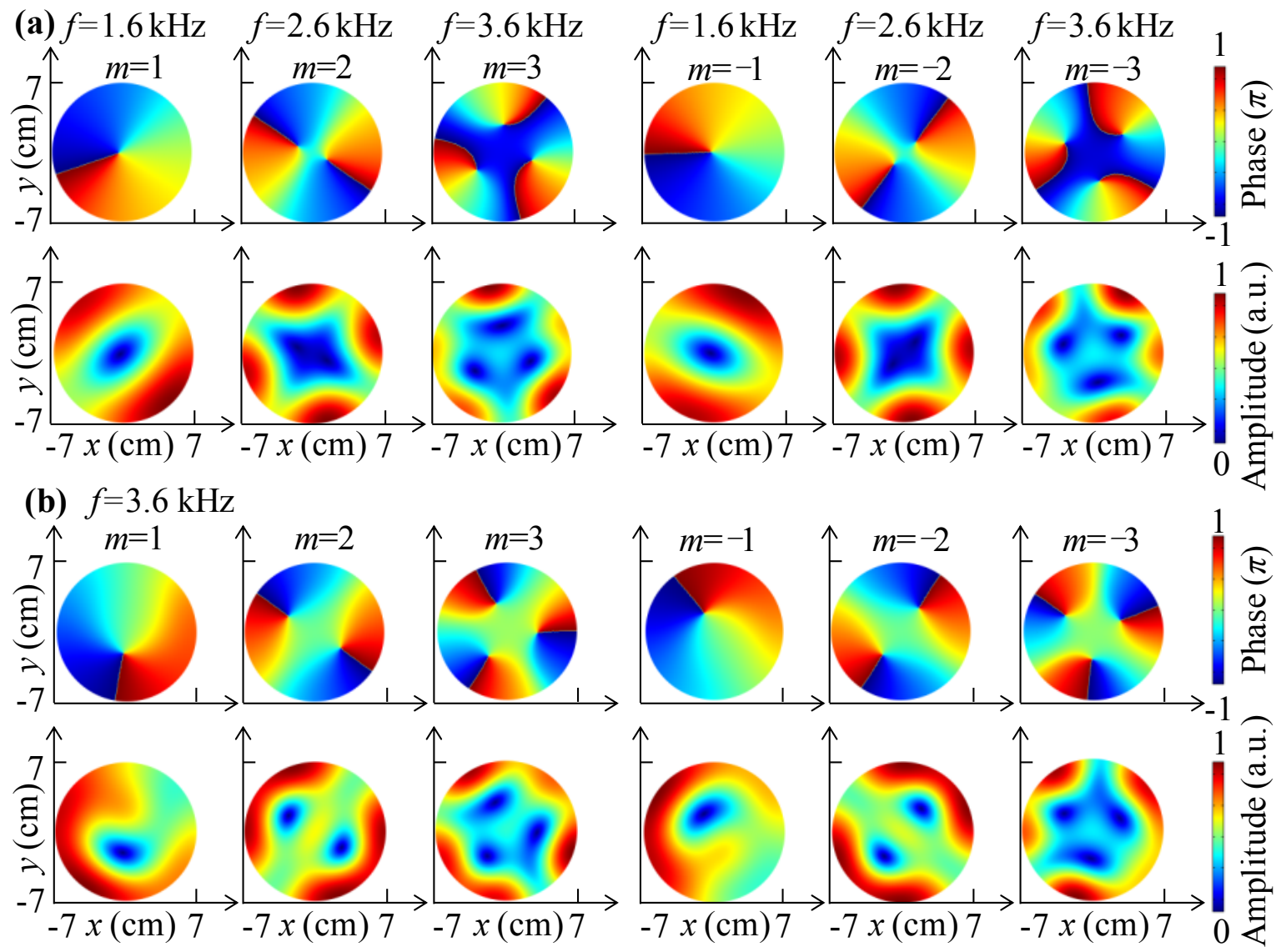

FIG. 3. (a) The reflected phase and amplitude distributions of the multiple order AVs with $|m|=l(l=1,2,3)$ at frequencies of 1.6, 2.6 and $3.6 \mathrm{kHz}$, respectively. (b) The phase and amplitude distributions at frequency of 3.6 $\mathrm{kHz}$.

First, the vortex transition from the near to the far field in the waveguide is concerned in the analysis and simulation, see supplementary material. In contrast, we discuss the limited propagation distance of the vortex in an infinite free space. It is found that the OAM pattern can propagate at 
most $2 \lambda$, where the pressure amplitude are reduced to indistinguishable, see supplementary material.

We also present the acoustic pressure fields including the amplitude and phase of the vortex using the thermoviscous acoustics model. The results indicate that the influence of the thermoviscous loss on the acoustic performances can be ignored (see supplementary material for details).

We then further systematically investigate the patterns of the multiple order AVs at different frequencies, as shown in Fig. 3. The reflected phase and amplitude distributions of the multiple order vortices with $|m|=l(l=1,2,3)$ at frequencies of $1.6,2.6$ and $3.6 \mathrm{kHz}$ are illustrated in Fig. 3(a), respectively. The observation transverse plane is located at $z=50 \mathrm{~cm}$. In the left side, it can be observed that the wavefront phases have changed $2 \pi, 4 \pi$ and $6 \pi$ in an annular loop of the phase fields. The corresponding pressure amplitude profile also has an approximate symmetric distribution with null pressure at the central region. Likewise, the phase variation of the multiple order vortices and the corresponding null amplitude in cores can be exhibited at a single frequency of $3.6 \mathrm{kHz}$ in the left side of Fig. 3(b). This cross-section is located at $z=60 \mathrm{~cm}$. In addition, although the helical channels of the TAM structure have a definite clockwise spiral direction, the reverse vortex fields with $m=-l$ $(l=1,2,3)$ can also be well formed in the three right-hand side panels of Fig. 3 . These all prove the generations of the first three order AVs. Note that the OAM mode propagation in the waveguide is the superposition of multiple Bessel modes especially at high frequencies. ${ }^{30}$ In addition, the sound waves reflected from the helical channels may not have a perfect plane wavefront and may be 
accompanied by the parasitic or high-order scattering. ${ }^{31}$ Thus, the null cores in some cases are not strictly located in the geometrical center or have a small asymmetry in the amplitude profiles.
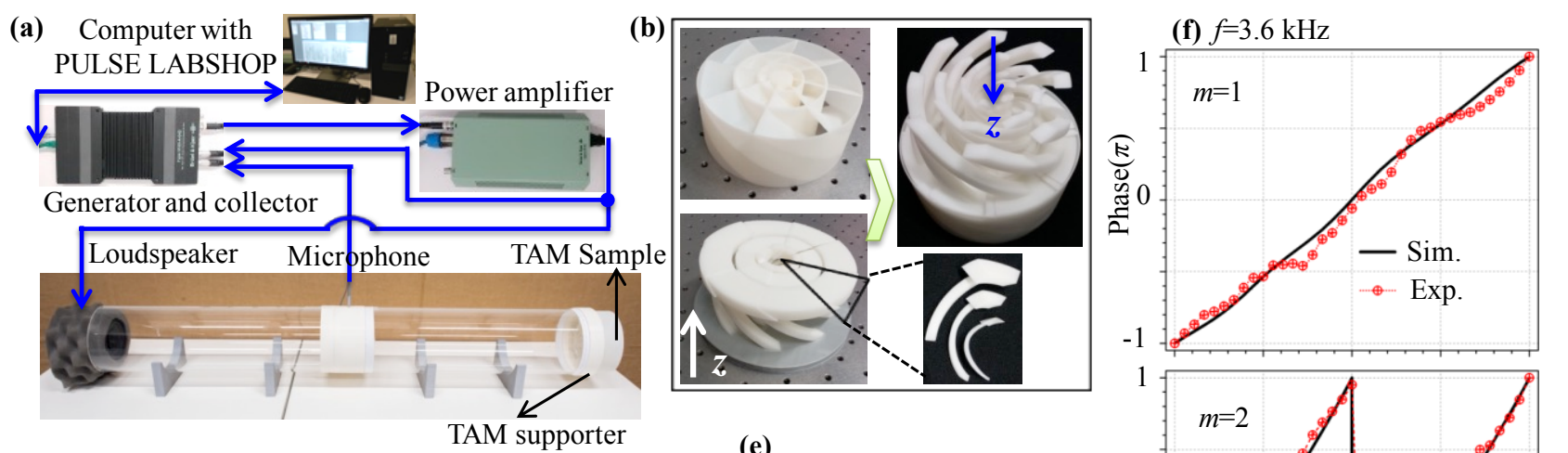

(c) $f=1.6 \mathrm{kHz}, m=1$
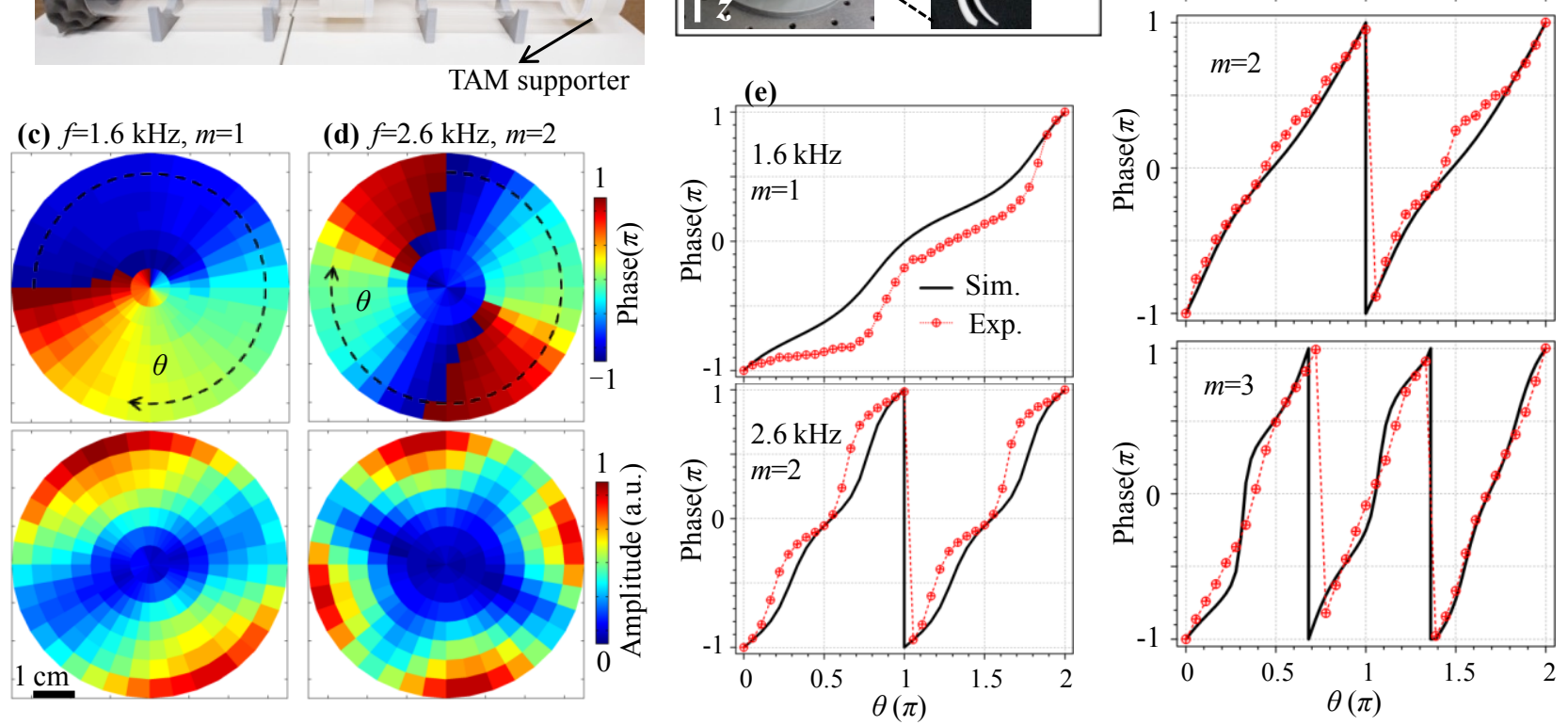

FIG. 4. (a) Schematic of the experimental setup. (b) Photographs of the metasurface sample. Experimental measurements of the phase and amplitude distributions for (c) the first-order vortex at frequency of $1.6 \mathrm{kHz}$ and (d) the second-order vortex at frequency of $2.6 \mathrm{kHz}$. (e) The variation of the phase values with the azimuthal angle for the cut lines of (c) and (d) in an annular loop at the radial position $r=6 \mathrm{~cm}$. (f) Experimental results for 1st-order, 2nd-order, and 3rd-order vortices at frequency of $3.6 \mathrm{kHz}$. 
Experimental measurements are designed and carried out to evidence the generation of the broadband tunable AVs by the proposed TAM. Figure 4(a) shows the schematic diagram of the experimental setup. The components of the TAM are fabricated with enough precision $(0.1 \mathrm{~mm})$ in Fig. 4(b). The details of the devices and the measurement processes are found in supplementary material. The experimental results for the first and second order vortices at the frequencies of 1.6 and $2.6 \mathrm{kHz}$ are shown in Figs. 4(c) and 4(d), respectively. Good agreements of the phase and amplitude profiles are observed between the simulations in Fig. 3 and measurements in Fig. 4. For quantitative comparison, the circular cut lines of the phase profiles at $r=6 \mathrm{~cm}$ in the radial position (dashed lines with arrow) are plotted in Fig. 4(e). The phases have shifted from $-\pi$ to $\pi$ for one time and two times in an annular loop for the first-order and second-order TAM configurations, which clearly shows that the TCs of the corresponding generated AVs are $m=1$ and 2, respectively. In addition, Figure 4(f) shows that both the simulated and the experimental phases have shifted $2 m \pi$ for the multiple order OAM modes at the single frequency of $3.6 \mathrm{kHz}$. This proves that the designed TAM possesses the characteristic of continuously tunable TC in the waveguide system.

To conclude, we have proposed a continuously tunable acoustic vortex generator based on a reconfigurable AM with theoretical analysis, numerical simulation, and experimental demonstration. The designed TAM is formed by the matched helical channels to modulate the reflected phase shifts of the individual units. This adjustable design is capable of spatially spiraling the acoustic wave over 
a wide frequency range and producing the twisted multiple order AVs in the waveguide system. Each vortex order at different frequencies and the multiple OAM models at a single frequency are realized by the reconfiguration of only one TAM. We expect that the TAM can yield AVs with remarkable flexibility and suggest exciting possibilities in a variety of applications. The latter include the real-time acoustic communication, micro-particles manipulation, and micro-fluidic fast mixing.

See the supplementary material for the propagable frequency range, the theoretically derivation, the interactions between units, the tuned helical depth, the vortex transition in the waveguide, the vortex in the free space, the influences of the thermoviscous loss effects and the experimental measurement in detail.

\section{Acknowledgments:}

This work is supported la Région Grand Est, by the Institut CARNOT ICEEL, and by the National Natural Science Foundation of China (Nos. 11872101 and 11991031). The first author is grateful for the support of the National Natural Science Foundation of China (No. 11502123), the Natural Science Foundation of Inner Mongolia Autonomous Region of China (No. 2015JQ01), and the China Scholarship Council (CSC No. 201807090043). 


\section{References}

${ }^{1}$ B. Assouar, B. Liang, Y. Wu, Y. Li, J. C. Cheng and Y. Jing, Nat. Rev. Mater. 3, 460 (2018).

${ }^{2}$ L. Cao, Z. Yang, Y. Xu, S. W. Fan, Y. Zhu, Z. Chen, B. Vincent and B. Assouar, Phys. Rev. Appl. 13, 014054 (2020).

${ }^{3}$ S. M. Yuan, A. L. Chen and Y. S. Wang, J. Sound Vib. 470, 115168 (2020).

${ }^{4}$ Y. F. Wang, Y. Z. Wang, B. Wu, W. Chen and Y. S. Wang, Appl. Mech. Rev. 72, 040801 (2020).

${ }^{5}$ Y. Li, C. Shen, Y. Xie, J. Li, W. Wang, S. A. Cummer and Y. Jing, Phys. Rev. Lett. 119, 035501 (2017).

${ }^{6}$ J. P. Xia, X. T. Zhang, H. X. Sun, S. Q. Yuan, J. Qian and Y. Ge, Phys. Rev. Appl. 10, 014016 (2018).

${ }^{7}$ Y. Zhu, F. Fei, S. W. Fan, L. Cao, K. Donda and B. Assouar, Phys. Rev. Appl. 12, 034029 (2019).

${ }^{8}$ G. Ma, X. Fan, P. Sheng and M. Fink, Proc. Natl. Acad. Sci. USA 115, 6638 (2018).

${ }^{9}$ Z. Tian, C. Shen, J. Li, E. Reit, Y. Gu, H. Fu, S. A. Cummer and T. J. Huang, Adv. Funct. Mater. 29, 1808489 (2019).

${ }^{10}$ S. D. Zhao, A. L. Chen, Y. S. Wang and C. Zhang, Phys. Rev. Appl. 10, 054066 (2018).

${ }^{11}$ A. L. Chen, Q. Y. Tang, H. Y. Wang, S. D. Zhao and Y. S. Wang, Sci. China: Phys., Mech. Astron. 63, 244611 (2020).

${ }^{12}$ S. W. Fan, S. D. Zhao, A. L. Chen, Y. F. Wang, B. Assouar and Y. S. Wang, Phys. Rev. Appl. 11, 044038 (2019).

${ }^{13}$ X. S. Li, Y. F. Wang, A. L. Chen and Y. S. Wang, Sci. Rep. 9, 15856 (2019).

${ }^{14}$ S. W. Fan, S. D. Zhao, L. Cao, Y. Zhu, A. L. Chen, Y. F. Wang, K. Donda, Y. S. Wang and B. Assouar, Phys. Rev. B 101, 024104 (2020).

${ }^{15}$ X. S. Li, Y. F. Wang, A. L. Chen and Y. S. Wang, J. Phys. D: Appl. Phys. 53, 195301 (2020).

${ }^{16}$ Z. Hong, J. Zhang and B. W. Drinkwater, Phys. Rev. Lett. 114, 214301 (2015).

${ }^{17}$ X. Jiang, B. Liang, J. C. Cheng and C. W. Qiu, Adv. Mater. 30, 1800257 (2018).

${ }^{18}$ C. J. Naify, C. A. Rohde, T. P. Martin, M. Nicholas, M. D. Guild and G. J. Orris, Appl. Phys. Lett. 108, 223503 (2016).

${ }^{19}$ W. Li, M. Ke, S. Peng, F. Liu, C. Qiu and Z. Liu, Appl. Phys. Lett. 113, 051902 (2018).

${ }^{20}$ Y. Li, G. Guo, J. Tu, Q. Ma, X. Guo, D. Zhang and O. A. Sapozhnikov, Appl. Phys. 
Lett. 112, 254101 (2018).

${ }^{21}$ A. Marzo, M. Caleap and B. W. Drinkwater, Phys. Rev. Lett. 120, 044301 (2018).

${ }^{22}$ X. Jiang, J. Zhao, S. L. Liu, B. Liang, X. Y. Zou, J. Yang, C. W. Qiu and J. C. Cheng, Appl. Phys. Lett. 108, 203501 (2016).

${ }^{23}$ N. Jiménez, V. Romero-García, L. M. García-Raffi, F. Camarena and K. Staliunas, Appl. Phys. Lett. 112, 204101 (2018).

${ }^{24}$ D. C. Chen, Q. X. Zhou, X. F. Zhu, Z. Xu and D. J. Wu, Appl. Phys. Lett. 115, 083501 (2019).

${ }^{25}$ Y. R. Jia, W. Q. Ji, D. J. Wu and X. J. Liu, Appl. Phys. Lett. 113, 173502 (2018).

${ }^{26}$ Y. Zhang, B. Xie, W. Liu, H. Cheng, S. Chen and J. Tian, Appl. Phys. Lett. 114, 091905 (2019).

${ }^{27}$ X. Jiang, Y. Li, B. Liang, J. C. Cheng and L. Zhang, Phys. Rev. Lett. 117, 034301 (2016).

${ }^{28}$ J. F. Zeng, X. Zhang, F. G. Wu, L. X. Han, Q. Wang, Z. F. Mu, H. F. Dong and Y. W. Yao, Phys. Lett. A 383, 2640 (2019).

${ }^{29}$ H. Esfahlani, H. Lissek and J. R. Mosig, Phys. Rev. B 95, 024312 (2017).

${ }^{30}$ Z. Guo, H. Liu, H. Zhou, K. Zhou, S. Wang, F. Shen, Y. Gong, J. Gao, S. Liu and K. Guo, Phys. Rev. E 100, 053315 (2019).

${ }^{31}$ L. Cao, Y. Xu, B. Assouar and Z. Yang, Appl. Phys. Lett. 113, 183506 (2018). 